\title{
Color-Based for Tree Yield Fruits Image Counting
}

\author{
Faisel G. Mohammed, Wejdan A. Amer \\ Remote Sensing \& GIS Dep., College of Science, University of Baghdad, faisel@scbaghdad.edu.iq \\ wejdan7449@gmail.com \\ Baghdad, Iraq
}

\begin{abstract}
- identifying the total number of fruits on trees has long been of interest in agricultural crop estimation work. Yield prediction of fruits in practical environment is one of the hard and significant tasks to obtain better results in crop management system to achieve more productivity with regard to moderate cost. Utilized color vision in machine vision system to identify citrus fruits, and estimated yield information of the citrus grove in-real time. Fruit recognition algorithms based on color features to estimate the number of fruit. In the current research work, some low complexity and efficient image analysis approach was proposed to count yield fruits image in the natural scene. Semi automatic segmentation and yield calculation of fruit based on shape analysis is presented. Color and shape analysis was utilized to segment the images of different fruits like apple, pomegranate obtained under different lighting conditions. First the input sectional tree image was converted from RGB colour space into the colour space transform (i.e., YUV, YIQ, or YCbCr). The resultant image was then applied to the algorithm for fruit segmentation. After it is applied Morphological Operations which is enhanced image then execute Blob counting method which identify the object and count the number of it. Accuracy of this algorithm used in this thesis is $82.21 \%$ for images that have been scanned.
\end{abstract}

Index Terms - image segmentation; object labeling; color space; fruit counting

\section{Introduction}

Computer image analysis largely contains the fields of computer or machine vision, and medical imaging, and makes heavy use of pattern recognition, digital geometry, and signal processing. Computers are indispensable for the analysis of large amounts of data, for tasks that require complex computation, or for the extraction of quantitative information [Abh14].

Computer vision is the science and technology of machines that see. As a scientific discipline, computer vision is concerned with the theory and technology for building artificial systems that obtain information from images or multi-dimensional data. Information is that which enables a decision [Mcc10].

Computer vision, on the other hand, studies and describes artificial vision systems that are implemented in software and/or hardware. Applications of computer vision systems include robots and autonomous vehicles, detection, organizing information, and modeling objects [Qin13].

To our knowledge, there has been automated system designed for kiwi fruit counting or any digital colour/gray image based automated counting system for counting kiwi fruit prior to harvesting. the method has been tested only on 20 trees and a validation result similar to our result has been achieved. This method has the disadvantage of being highly dependent on weather conditions. A recent work to quantify green apples in an orchard by using hyper spectral images and machine vision techniques [The14].

Fruit segmentation system was primarily developed for robotic fruit harvesting. However, this technology can easily be tailored for other applications such as on tree yield monitoring, crop health status monitoring, disease segmentation, maturity segmentation and other operations which require vision as a sensor.

We used the local or color based analysis for rapid fruit segmentation and was able to detect the fruit at specific maturity stages i.e., fruit with a color different from the background. The on-line estimation of oranges, peaches and apples regarding the quality attributes like size, color, stem location and segmentation of external blemishes was presented. 
Research and development in robotic tree fruit harvesting has been a major focus in recent years because of decreasing supply and increasing cost of human labor. One of the major tasks of a fruit harvesting robot is to identify or detect fruit in tree canopies. Machine vision-based fruit identification is also important for accurate crop-load estimation for crops such as apple and citrus [Ulz13].

\section{Related works}

Interest in machine vision of fruit has increased markedly between 1990 and 2012, particularly since 2004, as gauged by the number of publications listed by a Scopus search on keywords machine vision and fruit [Dut15].

1- In 2012, H. N. Patel [Pat12], efficient locating the fruit on the tree is one of the major requirements for the fruit harvesting system. In this paper, automatic segmentation and yield calculation of fruit based on shape analysis is presented.

2- In 2012, R. Zou et.al. [Ron12], introduced apple fruit recognition algorithms based on colour features are presented to estimate the number of fruits and develop models for early prediction of apple yield.

3- In 2012, U. Dorj [Ulz13] introduced a new counting algorithm for tangerine yield estimation is adapted to obtain better results with respect to partially / semi partially occluded tangerine and its clusters. To optimize tangerine counting, and to minimize typical background noises from orchards.

4- (Anisha Syal 2013) count the number of fruits on trees and their Yield estimation is performed. The various computer vision and optimization techniques are presented to automate the process of counting fruits [Ani13].

5- (Payne and K. Walsh 2014) A machine vision count of fruit based on images of trees of the entire orchard should allow better estimation than the current method, which is based on visual estimation of load on a small subset of trees in the orchard. Further, this application can tolerate a higher error in fruit location than a number of other applications, such as harvesting [Pay14].

\section{Problem Statement}

The problem of segmenting agricultural fields in aerial images is still a manual work in most Geographic Information System requiring repetitive, tedious and time consuming human work.

Address the problem of semiautomatic segmenting agricultural fields by region competition technique that integrates region growing and deformable models. The deformable model dynamically adapts its contour analyzing homogeneous parcels in an energy-minimizing framework.

Our work presents an automatic segmentation and calculation of yield fruit based on the color analysis. The color transformation was utilized to do the segmentation of the fruits in an input sectional tree image. The tested images used in current research work. The input color image was first converted from the RGB color space into the YCBCR color space for the coarse detection of fruit region. The YCBCR color space has been designed to resemble the human visual perception. The idea was to do the coarse processing of the image so that the fruit were visually well distinguishable and then to use the YCBCR color space to segment fruit regions with its perceptually uniform property. (i.e. the colors which are visually similar are close to each other in color space).

The edges were detected from the resultant image using edge detector. These edge points were used for fitting the nearest circular shape. The number of circles fitted to the input image was used as a count value of the fruits in an input image. 


\section{Proposed System}

Our work in this thesis presents an automatic segmentation and yield calculation of fruit based on shape analysis. The color and shape analysis was utilized to do the segmentation of the fruits in an input sectional tree image. The images used in our work were of different tree images of variety of fruits like apple, pomegranate, orange, peach, litchi and plum. The input color image was first converted from the RGB color space into the YCBCR color space for the coarse detection of fruit region. The YCBCR color space has been designed to resemble the human visual perception. The idea was to do the coarse processing of the image so that the fruit were visually well distinguishable and then to use the YCBCR color space to segment fruit regions with its perceptually uniform property. (i.e. the colors which are visually similar are close to each other in color space).

The edges were detected from the resultant image using edge detector. These edge points were used for fitting the nearest circular shape. The number of circles fitted to the input image was used as a count value of the fruits in an input image. [Sil15].



(a) Image 1

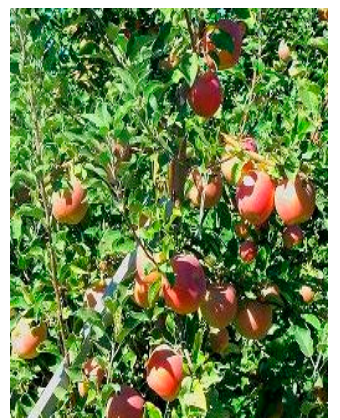

(b) Image 2

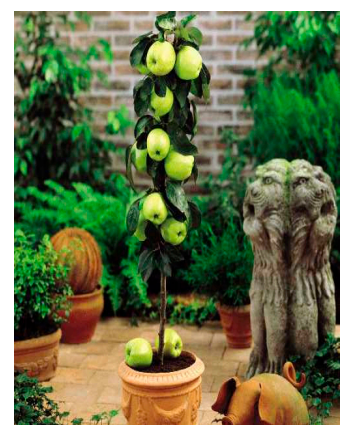

(c) Image 3

Fig. 1: Original Tested images have 24 bit/pixel

\subsection{Procedure Steps}

1. Find Min and Max gray levels for all pixels within image to calculate Range= [Max-Min].

2. Force all pixels to a new "stretched" value using the following formula: New Gray $=255 \times$ ((Old Gray - Min) / Range).

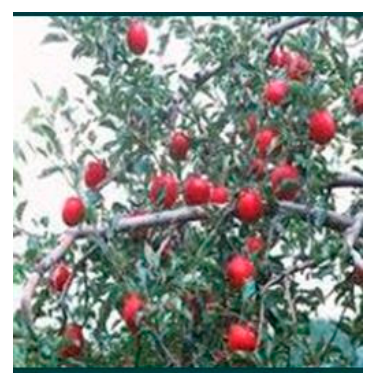

(a): before ACS

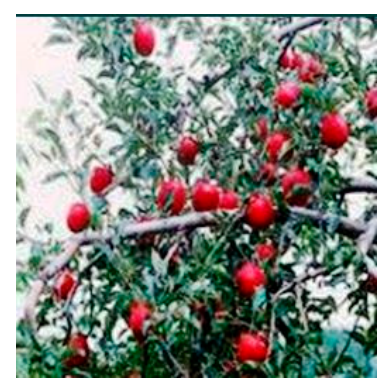

(b): after ACS

Fig (2): Image ACS 
3- Fruits isolated from the rest of the details of the image by using the segmentation technique and rely on color through the application of this requirement:

$$
\text { Output }_{\text {img }}= \begin{cases}1 & \text { if }(\text { Red component }-(\text { Blue component }+ \text { Green component })>100) \\ 0 & \text { Otherwise }\end{cases}
$$

Where 1 represent fruit pixel, while $\mathbf{0}$ represent Background


Fig (3): Segmentation using RGB color model.

5- Improve the parts of the image after segmentation process that was small and needed to be clarified are through dilation process by the application of this equation: (Image Morphological operations was showed in figure 4).

$$
\text { Output }_{\text {img }}=\left\{\begin{array}{lc}
1 & \text { if } s \text { hits } \mathrm{f} \\
0 & \text { Otherwise }
\end{array}\right.
$$



(a): Image after dilation,



(b): Image after Erosion

Fig (4): Image Morphological operations

or if they are large minimized and hide the track that were not fruit through erosion process by the application of this equation:

Output $_{i m g}= \begin{cases}1 & \text { if } s \text { fits } \mathrm{f} \\ 0 & \text { Otherwise }\end{cases}$ 
6 - The proposed method labels each component using fast and low complexity contour tracing technique. This method is based on the principle that a component is fully determined by its contours. This method also provides a procedure for finding all component pixels. An image will scan in the same way as it would be encountered by a scanner, i.e., from top to bottom and from left to right per each line.

\subsection{Object labeling}

When an external or internal contour is encountered, then the contour-tracing procedure will use to complete the contour and assign a label, say L, to all pixels on the contour. When the contour is traced back to its starting point, scanning will resume at that point. Later on, when the contour pixels labeled $\mathrm{L}$ are visited again, then the same label $\mathrm{L}$ will assign to black pixels that lie next to them. This method is applicable in areas in which must detect components and also classify them by means of certain contour features. The used labeling method was tacked from [Fai13]. Figure 5 show the final results.

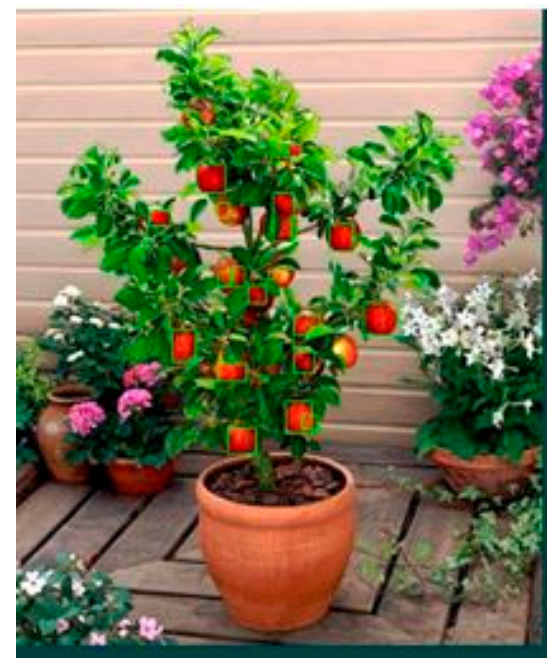

Fig (5): Object labeling: number of fruit are 20

\section{Results and Discussion}

To validate the proposed fruit detection algorithm, twenty digitalized images of fruits (apple) each contains more fruits. The detection algorithm proved to be successful as it missed or misclassified only 1 to 3 fruits per tree, where by the fruit detection was accurate with $82.21 \%$ recognition rate accuracy and an overall error rate $17.78 \%$. The accuracy of the fruit calculation was calculated based on the difference between the manual fruit count and fruit count using this algorithm as shown in the table 1 below. 
In this table, we have selected a set of images in order to work higher and we started counting the number of fruits and manually recorded the number of fruits and then we let the system calculate the number of fruits and after that we have made in the equation to find the difference between the two accounts and knowledge of the system's accuracy.

Table 1: System counting accuracy

\begin{tabular}{|c|c|c|c|c|}
\hline Image name & $\begin{array}{c}\text { Manual } \\
\text { count }\end{array}$ & $\begin{array}{c}\text { System } \\
\text { count }\end{array}$ & $\begin{array}{c}\text { Error rate(difference/ } \\
\text { Manual count *100\%) }\end{array}$ & $\begin{array}{c}\text { Accuracy } \\
\text { (100-error) }\end{array}$ \\
\hline Image 1 & 18 & 23 & $(5 / 18 * 100 \%)=27.78 \%$ & $72.22 \%$ \\
\hline Image 2 & 14 & 12 & $(2 / 14 * 100 \%)=14.28 \%$ & $85.72 \%$ \\
\hline Image 3 & 11 & 12 & $(1 / 11 * 100 \%)=9.09 \%$ & $90.91 \%$ \\
\hline Image 4 & 10 & 12 & $(2 / 10 * 100 \%)=20 \%$ & $80 \%$ \\
\hline & & & $\begin{array}{c}\text { Average error rate } \\
=17.78 \%\end{array}$ & $\begin{array}{c}\text { Average accuracy } \\
=82.21 \%\end{array}$ \\
\hline
\end{tabular}

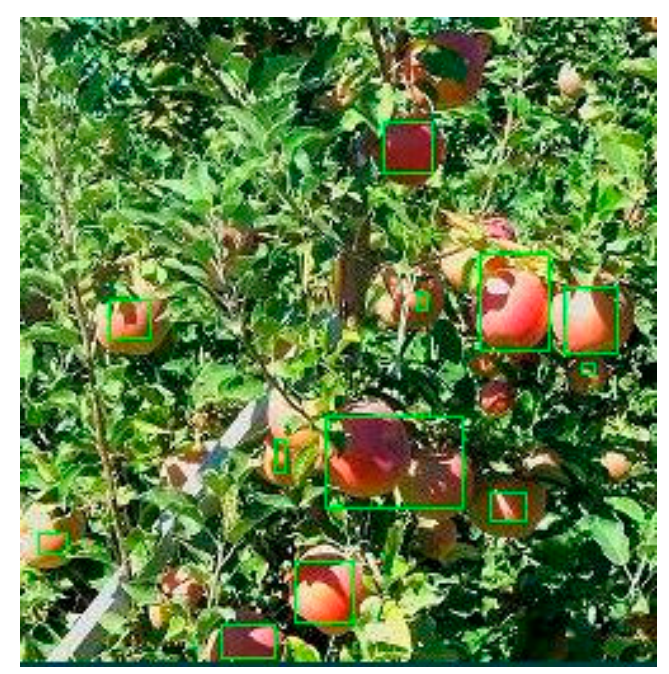

Fig 9: Result of proposed algorithm (Total no. of Fruits are 12 objects)

The proposed algorithm detects the fruits accurately in the different lighting conditions, shadow effects of leaves on fruit and in clustered background. It should be noted that there were very few over estimations and few under estimations by the algorithm 


\subsection{Reasons for overestimation were}

1. When a single fruit was hidden by many leaves and the separation was more than $9(3 \times 3)$ pixels, they were counted as different fruits.

2. Small fruits were not clearly visible in manual counting; however they were counted as fruits by the algorithm.

\subsection{Reasons for underestimation were}

In some cases, many fruits clusters were counted as single fruit in the estimation by the algorithm due to connectivity. The algorithm was able to detect the fruits in 4 of 40 images. In other words, the accuracy of the algorithm was found to be $82.21 \%$. The accuracy of the fruit calculation was calculated based on the difference between the manual fruit count and fruit count using algorithm. The fruits were calculated manually from an input image by two different persons. The average manual count was compared with the count resulted from an algorithm. The percentage error was the measure of the yield calculation accuracy. The plot of fruit count using algorithm versus manual count is shown in blew figure (3.9).

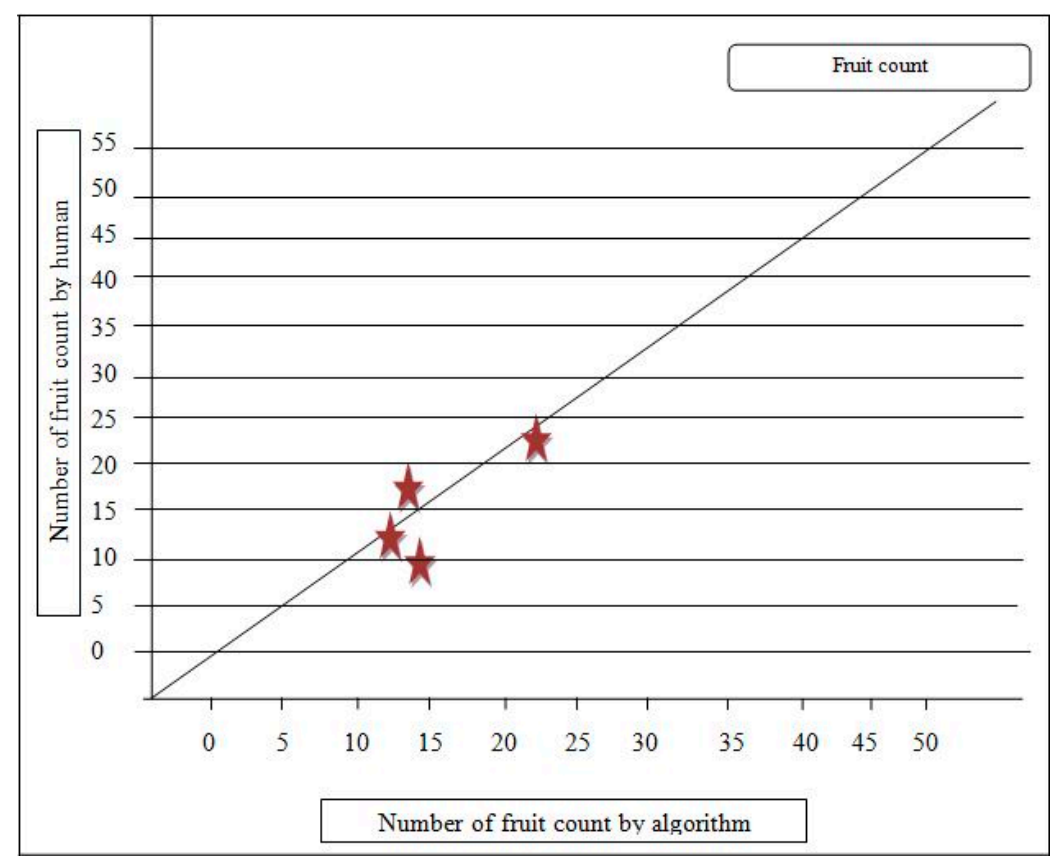

Fig (3. 9): Linear regression between algorithm and manual count

\subsection{Conclusion}

The automatic segmentation and yield measurement of fruit was developed and evaluated. The algorithm was composed region labeling based color detection. It was designed to solve the 
problems of varying illumination and fruit occlusion through segmentation and color-based detection.

So we can identify the fruit through colors and after work processes necessary we can separate the fruit from the rest of the image details and identified and then calculate the number of fruits in the picture under the lighting different, because we have made a choice to improve the image in terms of lighting and then we were able to improve the parts of the image after work retail her through Morphological operations and therefore we were able to calculate the number.

Thus, we can use the computer and machine vision to identify fruit and under different conditions and implementation of the operations, and ultimately get the desired result, which is in this work count the number of fruits.

\subsection{Future Suggestions}

There are some future works should be implement on the Fruits Recognition System in order to improve and enhance the functionality and flexibility of the recognition system for more widely usage.

- The system should be improved by extending its functions to process and recognize more variety of different fruit images. Besides that, the texture based analysis technique could be combined with the existing three features analysis technique on the system in order to gain better discerning of different fruit image.

- Can use a closed system for image acquisition, with a high definition camera i.e. CCD, this might help to avoid the grain shadow observed in the database acquired and will also give uniformity in the intensity of background color.

- The present work could be extended for remaining fruit also and few other features can also be extracted to increase accuracy. Also various infections on fruit like fissures can also be identified further.

\section{References}

[Abh14] Abhisesh Silwal, Aleana Gongal, Manoj Karkee, "Identification of red apples in field environment with over the row machine vision system", Agric Eng Int: CIGR Journal Open access at http://www.cigrjournal.org, Vol. 16, No. 4, 2014.

[Ani13] Anisha Syal, "A Survey of Computer Vision Methods for Counting Fruits and Yield Prediction1Student, CSE Department, ASET, Amity University, 2013.

[Fai13] Faisel G. Mohammed, "An efficient multiple pass pixel connectivity labeling method for object detection", International Journal of Scientific \& Engineering Research, Volume 4, Issue 5, May-2013.

[Mar14] Maria P Diago, "Assessment of cluster yield components by image analysis", published online in Wiley Online Library, 2014. 
[Mcc10] C. L. McCarthy N. H. Hancock S. R. Raine, "Applied machine vision of plants - a review with implications for field deployment in automated Farming operations", Author's Accepted Version of McCarthy, University of Southern Queensland, 2010.

[Pat12] H. N. Patel, "Automatic Segmentation and Yield Measurement of Fruit using Shape Analysis", Associate Professor, A.D. Patel Institute of Technology, 2012.

[Pay14] A. Payne and K. Walsh, "chapter 16. Machine vision in estimation of fruit crop yield",Plant Image Analysis: Fundamentals and Applications ,Pages 329-374, CRC Press 2014.

[Qin13] Qin Zhang, WSU Center for Precision \& Automated Agricultural Systems, Washington State University, 2013.

[Ron12] Rong Zhou, Lutz Damerow, Yurui Sun \& Michael M. Blanke, "Using colour features of cv. 'Gala' apple fruits in an orchard in image processing to predict yield", An International Journal on Advances in Precision Agriculture, 2012.

[Sil15] P.F. Silva,"Development of a quantitative semi-automated system for intestinal morphology assessment in Atlantic salmon using image analysis", Skretting Aquaculture Research Centre, 2015.

[Son13] Y. Song, "Automatic fruit recognition and counting 1 from multiple images", Biomathematics and Statistics Scotland, Edinburgh, EH9 3JZ, UK, 2013.

[The14] Theofanis A. Gemtos, Spyros Fountas, Katerina Aggelopoulou, "Precision Agriculture Applications in Horticultural Crops in Greece and Worldwide", University of Thessaly, 2014.

[Ulz13] Ulzii-Orshikh Dorj," A Counting Algorithm for Tangerine Yield Estimation", 1Center for Advanced Image and Information Technology, School of Electronics \& Information Engineering, Chon Buk National University, 2013.

[Ulz13] Ulzii-Orshikh Dorj1, Malrey Lee, and Sang sub Han, "A Counting Algorithm for Tangerine Yield Estimation", 1Center for Advanced Image and Information Technology, School of Electronics \& Information Engineering, Chon Buk National University, 2013. 